Technologie des Ubiquitous Computing erlaube, dass die Dinge über uns informiert sind bzw. uns steuern. Dabei ist für den Einzelnen nicht immer feststellbar, warum gerade was geschieht. „Richtig gefährlich wird es, wenn die Dinge in strategischer Absicht nicht nur das Handeln der mit ihnen interagierenden Subjekte steuern, sondern eine Koordination des Handelns Vieler herbeiführen“, so Hubig.

Er fordert eine „Parallelkommunikation“, die den Nutzern die Kriterien transparent macht, nach denen die Gegenstände agieren. Darüber hinaus sollte der Nutzer stets die Möglichkeit haben, einzugreifen, die Handlung zu beeinflussen oder die Nutzung zu verweigern. Der Mensch - so sein Credo - muss als selbstständig handelndes Subjekt erhalten bleiben und nicht zum Objekt der agierenden Dinge werden.

Ein Problem des mobilen Arbeitens und Lebens liegt bereits bei dem heutigen Stand der Technik im Datenschutz. Wie soll das erst in einer Welt allgegenwärtiger vernetzter Computer werden? Claudia Eckert, Professorin für Sicherheit in der Informationstechnik an der TU Darmstadt, pochte in Berlin auf Datenschutz in der ,schönen neuen Welt“. Natascha Adamowsky ging erst gar nicht auf solch konkrete Probleme ein; für sie ist die Revolution durch die Computertechnologie in Gestalt des Ubiquitous Computing bereits vorprogrammiert: Die Professorin für Kulturwissenschaft an der Humboldt-Universität zu Berlin forderte eine ernsthafte Diskussion über Sinn und Zweck, Inhalt und Form des Ubiquitous Computing ein. Entscheidend für das Leben in einer Welt smarter Alltagsdinge sind schließlich die konkreten Projekte, so Adamowsky. Sie möchte, dass endlich über die Projekte und ihre Sinnhaftigkeit geredet wird, während sie bereits Gestalt annehmen.

Noch nicht morgen, aber übermorgen: Für Friedemann Mattern, den Initiator und Leiter des 7. Berliner Kolloquiums der Gottlieb Daimler- und Karl Benz-Stiftung, ist der Zeithorizont zur Realisierung der Vision einer total vernetzten, informatisierten Welt mittelund langfristig angelegt. Bei der autonomen Energieversorgung, aber auch bei der Realisierung eines anpassungs- und lernfähigen Verhaltens „smarter“ Dinge steht die Forschung zwar noch am Anfang. Friedemann Mattern geht jedoch davon aus, dass die Vision einer umfassenden Informatisierung der Welt und ihrer Dinge in den nächsten Jahren tatsächlich realisierbar sein wird - jedenfalls aus technischer Sicht.

Weitere Informationen im Internet unter http://www.smart-environment.de/berlin.html oder direkt im bereits erschienenen Tagungsband ,Total vernetzt - Szenarien einer informatisierten Welt", Friedemann Mattern (Hrsg.), Springer-Verlag Berlin Heidelberg 2003, ISSN 1439-5428

\section{Möglichkeiten und Grenzen der Verkehrstelematik}

\section{Bericht von der Öffentlichen Tagung der TA-SWISS am 15. April 2003 in Bern}

\section{von Ekkehard Fulda, Karlsruher Forum Ethik in Recht und Technik}

Unter dem Motto „Osterstau ohne Ende?“ wurden auf der Tagung im Kommunikationsmuseum in Bern zunächst die Hauptergebnisse der Studie „Das vernetzte Fahrzeug. Verkehrstelematik für Strasse und Schiene" vorgestellt, die das Zentrum für Technologiefolgen-Abschätzung TA-SWISS und das Bundesamt für Strassen (ASTRA) zur Verkehrstelematik (VT) erarbeitet hatten (Arbeitsdokument TA-DT 33/2003; siehe auch den Bericht in der TA-TuP in Heft 1/2003, S. 111 ff.). Die Schlussfolgerungen dieser TA-Untersuchung für die verschiedenen im Verkehrsgeschehen involvierten Adressaten wurden auf der Veranstaltung diskutiert, besonders solche zum Road-pricing.

Der Geschäftsführer der TA-Swiss, Sergio Bellucci, betonte in seiner Einführung die sowohl unabhängige als auch beratende Rolle seiner Institution, die nicht selbst Entscheidungsträger ist, sondern, analog zum Büro für Technikfolgen-Abschätzung beim Deutschen Bundestag (TAB), ihren Forschungs- und Beratungsauftrag vom Bundesparlament erhält.

Die Projektleiterin K. Schneeberger veranschaulichte an drei ausgewählten verkehrstelematischen Praxis-Versuchen in der Schweiz 
zentrale Funktionen von Verkehrstelematik: Die Verteilung und Dosierung von Verkehren wurde am Beispiel des „Tropfen-Zählers“ beim neu hergerichteten Gotthardtunnel erläutert, mit dem der Lastwagen-Verkehr gesteuert wird. Der Vernetzung von individuellem und öffentlichem Verkehr dient das Projekt „Car-los“ in der Region Burgdorf im Kanton Bern. Die dritte Hauptfunktion der Verkehrstelematik, die erhöhte Sicherheit, wurde am Bereich der Schieneninfrastruktur mit Lok-Steuerung exemplifiziert, wobei, neben den aktuellen Daten des Fahrzeuges selbst, von außen übertragene Daten das Fahrverhalten beeinflussen, bis hin zur automatischen Zugführung.

Der Leiter der Abteilung Strategie und Forschung des Bundesamtes für Strassen (ASTRA, Bern) Andreas Gantenbein, betonte das Erfordernis, die Verkehrspolitik und die Verkehrstelematik als Instrument der Verkehrspolitik deutlich $\mathrm{zu}$ unterscheiden; zu leicht würden sonst bestimmte VT-Anwendungen mit bestimmten, keinesfalls allgemein gutgeheißenen Zielen verwechselt, was oft die politische Akzeptanz erschwere. Er warnte, manche Möglichkeiten der Verkehrstelematik gingen weiter als das, was ,in der schweizerischen Politik als tragfähig gelten kann". Insbesondere seien noch eine Reihe von Rahmenbedingungen bzw. Grundlagen der Einführung von Verkehrstelematik herzustellen. Im „Leitbild“-Entwurf der Schweizer Verkehrspolitik habe es manche Idee gegeben, „die sich politisch kaum als mehrheitsfähig“" erwiesen hätte. Der „Sachplan Strasse“ räume der Verkehrstelematik hohe Bedeutung ein und schenke ,der Zusammenarbeit aller beteiligten Akteure (Bund, Kantone, Städte, Gemeinden, Polizei, Verkehrsteilnehmer, Dienstanbieter, Gerätehersteller, Planer und Systemlieferanten) hohe Beachtung". Gemäß schon bestehender gesetzlicher Regelungen (53 a, 57 c SVG) könne der Bund VT-Systeme auf den „bundesweit relevanten Straßen" einführen.

Nach den Ausführungen des Leiters der Vorentwicklung Mercedes-Benz Lkw, DaimlerChrysler (Stuttgart), Jürgen Trost ist eine weitere Steigerung der Verkehrssicherheit bei Lkw nicht mehr mit herkömmlichen Sicherheitssystemen zu erreichen. Nur mit neuartigen Systemen, die auch Informationen aus dem Umfeld des Fahrzeuges zu berücksichtigen in der Lage sind, seien noch signifikante Verbesserun- gen der Verkehrssicherheit zu erzielen. Dazu gehörten Systeme, die über eine eigene Sensorik verfügen, Systeme, die mit der Infrastruktur kommunizieren können sowie Systeme mit Kommunikation zwischen Fahrzeugen. Der Schwerpunkt der Entwicklung von MercedesBenz Lkw liege bei Systemen mit Sensorik, anhand der sich Unfälle auch dann vermeiden ließen, „wenn der Fahrer vorübergehend seine Verantwortung für das Fahrzeug nicht wahrnehmen kann". Die Probleme, derartige Systeme zur Erhöhung der Sicherheit einzuführen, liegen nach Einschätzung von Jürgen Trost nicht mehr im technischen Bereich, sondern an der zu geringen Nachfrage. Sogar die weitere Entwicklung der Systeme sei gefährdet, obwohl doch die weiter zunehmende Verkehrsdichte und der „massive wirtschaftliche Druck, unter dem die Branche der Transportdienstleister steht", die Einführung der neuen Sicherheitssysteme gerade erforderlich machten. Als Resümee forderte Trost (indirekt) staatliche, und zwar internationale, Maßnahmen zur flächendeckenden Einführung der neuen VT-Sicherheitssystemen an schweren Lkw: „Nur wenn ein gesellschaftlicher Konsens über den Einsatz neuartiger Sicherheitssysteme und deren Rahmenbedingungen erreicht werden kann, werden die Ziele des EUWeißbuchs - Reduzierung der Unfalltoten bis zum Jahre 2010 um die Hälfte - erreichbar.“

Die Diskussion (Leitung: Fulvio Caccia, Präsident der ComCom, Bern) behandelte die Rolle wirtschaftlicher Anreize zur Einführung VT-gestützter Sicherheitssysteme. Der Vertreter der Güterverkehrs-Wirtschaft, Fridolin Landolt (Planzer Transport AG, Dietikon) wies auf die Bedeutung von reduzierten Versicherungsprämien hin, die für entsprechend ausgestattete Lkw gewährt werden. Dagegen betonte Trost, dieses Anreizsystem bliebe zu schwach. Der Vertreter der Wissenschaft, Franz Mühletaler (ASIT, Bern) richtete den Blick auf den „gesellschaftlichen und politischen Rahmen“, der unverzichtbar dafür sei, dass die Sicherheitssysteme, die längst zur Einsatzreife gebracht sind, auch eingesetzt würden. Über die Sensorik-Systeme hinaus sollten auch Systeme mit Fahrzeug-zu-Fahrzeug-Kommunikation entwickelt werden.

Der Schweizer Datenschutzbeauftragte, Kosmas Tsiraktsopoulos, stellte bestehende Konflikte dar, die zwischen dem Erfordernis 
von Lenkungsaufgaben und Sicherheitsfragen einerseits und dem Datenschutz wie auch der individuellen Freiheit andererseits bestehen. Sehr deutlich wurde diese Situation angesichts der Alternative etwa in der Frage: Wie viel Verzicht auf individuelle Freiheit z. B. für einzelne Fahrzeuglenkende sind wir bereit in Kauf zu nehmen, um die Zahl der Verkehrstoten auf Null zu bringen? Werden Lokführer ,entmündigt", wenn das Sicherheitssystem auf vorübergehenden Verlust der Aufmerksamkeit mit Zugstopp reagiert; oder wenn ein System im Pkw eine Übermüdung der lenkenden Person durch mehr oder weniger penetrante Warnsignale anzeigt? Auch gravierende Probleme für den Datenschutz seien bei etlichen VTSystemen vorprogrammiert, ohne dass potenzielle Nutzer oder etwa Politiker sich ihrer bewusst seien. Derartige Zielkonflikte seien aber nicht unbedingt technisch, organisatorisch oder etwa allein in Expertendiskussionen lösbar. Vielmehr komme es darauf an, sie zu erkennen, um, nach angemessener Diskussion, durch politische Entscheidungen die entsprechenden Regelungen für den Umgang mit ihnen zu treffen. Die Diskussion widmete sich auch der Frage, ob neben schweren Lastwagen auch alle Pkw mit gegen Manipulation weitgehend gesicherten Black-boxes, analog zu Flugzeugen, ausgestattet werden sollten, auch wenn sie mit Datenschutzproblemen behaftet sein mögen. Eine generelle präventive Funktion derartiger Black-boxes wurde allerdings auch entschieden bestritten (Rudolf Zumbühl, TCS).

Der Schweizer Bundesrat Peter Vollmer plädierte engagiert für die erforderlichen Investitionen in Sicherheitssysteme und für entsprechende Rahmensetzung von staatlicher Seite, um deren generelle Verbreitung durchzusetzen. Dies sei wie beim seinerzeitigen Unterfahrschutz bei Lastwagen, der auch nur gegen zähe Lobbyarbeit der Transportunternehmen habe durchgesetzt werden können. Vertreten wurde in der Diskussion auch, dass VTSysteme zur erhöhten Sicherheit, z. B. auf der Schiene wie das ETCS - European Train Control System, auch mit deutlich verbesserter Effizienz einhergehen könnten (Peter Zoche, Fraunhofer ISI, Karlsruhe). Die Situation des Güterverkehrs steht in der Schweiz, wie Bundesrat Vollmer verdeutlichte, im Gegensatz zu der in Deutschland, denn beim Alpentransit sei die Bahn dominierend. Dies sollte angesichts weiter steigender Verkehrsaufkommen und Verkehrsdichte mittels VT-Systemen noch ausgebaut, die Bahn effektiver gemacht werden.

Der zweite Teil der Tagung thematisierte das „Road pricing“. Gilbert Saby, Directeur Général der Société marseillaise Tunnel Prado Carénage (SMTPC), berichtete über die Bauentscheidung sowie Planung und Realisierung des Tunnels. Dieser verlängert den unter dem Vieux Port verlaufenden Tunnel St. Laurent, und verbindet dessen Südufer (Quai de Rive Neuve) mit Prado weiter im Südosten, wo die Autobahn in östlicher Richtung nach Aubagne angebunden ist. Mit dem Projekt sollte die südliche Innenstadt, namentlich die Altstadt am Alten Hafen, vom Durchgangsverkehr entlastet, und damit auch die dortige Umweltsituation verbessert werden. Die der Stadt entstehenden Kosten aber waren so gering wie möglich zu halten. Von der politischen Entscheidung bis zur Eröffnung vergingen nur fünfeinhalb Jahre (1988-1993), die Bau- und Betriebsgesellschaft erhielt das Recht, auf 32 Jahre Nutzungsgebühren zu erheben. Nach Sabys Bericht harmonieren bei dem Projekt die Interessen der Kommune mit denen der Tunnelgesellschaft; denn die SMTPC sei in wirtschaftlicher Hinsicht an möglichst vielen Kunden interessiert, wodurch die Entlastungsfunktion ebenfalls gut erfüllt würde. Es handelt sich in Frankreich um den ersten Fall einer städtischen Straßenmaut, weshalb besonders darauf geachtet wurde, deren Höhe akzeptabel zu halten. Die Mautgebühr sollte von den potenziellen Nutzern als ein angemessener Preis für Zeitgewinn, Komfort und Sicherheit aufgefasst werden. Von Anfang an seien ,schmerzlose Zahlungsmodalitäten" in Form von Bankkarten und Télé péage realisiert worden, auch Möglichkeiten anonymer Zahlung. Das Projekt sei erfolgreich; in dem runden Jahrzehnt seines praktischen Betriebes mit über 100 Mio. Durchfahrten habe es keinen Protest gegen die Nutzungsgebühren gegeben.

Der Verkehrsexperte Thierry $d u$ Crest (CERTU, Lyon/Centre Bus Flandre, Pantin) analysierte die Road-pricing-Systeme von Marseille, Singapur und London. Seiner Darstellung zufolge stellen sich beim Road-pricing in städtischen Arealen ,heutzutage keine technischen, sondern nur noch politische Fragen“". Den politi 
schen Zielfestlegungen - abzuwägen zwischen: Finanzierung der Verkehrsinfrastruktur, Verkehrsentlastung, Umweltentlastung - lasse sich eine geeignete Kombination von Instrumenten zur Zielerreichung zuordnen. In Singapur habe man inzwischen ein barrierefreies elektronisches Zahlungssystem eingeführt, gleiches gelte für das im Februar 2003 eingeführte Londoner Modell. Die angestrebten Steuerungswirkungen für den Verkehr und die Umweltentlastung würden gut erfüllt. Bei den betrachteten Modellen werde auch das Ziel einer zusätzlichen Finanzierungsquelle für die Städte befriedigend erreicht. In London beläuft sich die Gebühr auf 5 Pfund pro Tag; in Singapur je nach vorgegebenem Steuerungsziel zwischen 0,3 und 1,3 Euro pro Fahrt in den bepreisten Bereich. Dort findet alle drei Monate eine Auswertung und Neufestlegung der Maut statt, die sich am verkehrlichen Ziel orientiert, eine Fahrgeschwindigkeit von $20-30 \mathrm{~km} / \mathrm{h}$ zu erzielen. Wie du Crest resümierte, kommt es bei der Umsetzung derartiger Mautprojekte vor allem auf die politische Kommunikation und die ausgehandelten Absprachen an.

In der Diskussion hob Bundesrat Peter Vollmer die sehr gute Lenkungswirkung des Road-pricings für den Individualverkehr hervor, ebenso die gute Eignung als Finanzierungsquelle für die Verkehrsinfrastruktur. Vor allem könne damit die Belastung verursachergerecht gestaltet werden: „Für mich ist es die Zukunft!“ Dagegen vermisste der Präsident des Schweizer Touring Clubs, Rudolf Zumbühl, an den Roadpricing-Systemen das Element der Angebotssteuerung; Road-pricing reguliere ja ausschließlich die Nachfrage. Politisch sei fragwürdig, dass es sich beim Road-pricing letztlich um ein undurchschaubares "Gemisch von Zielen“ handele. Der Schweizer Bundes-Datenschützer warnte nachdrücklich vor der ,Schwachstelle Mensch"; man dürfe nicht zu viele Daten sammeln. Er schlug vor, immer auch anonyme Alternativen beim Zahlen anzubieten, wie in Marseille beispielsweise mit Telefonkarten. Der Vertreter der Güterwirtschaft blieb insgesamt noch skeptisch: Die Erfahrungen mit bisherigen Schwerlastabgaben-Systemen hätten gezeigt, dass die Technik noch keineswegs stabil funktioniere; die Qualität der automatisch erhobenen Daten ließe noch zu wünschen übrig.

\section{FutureScene 2003}

\author{
St. Paul de Vence, France, April 14 - 15, \\ 2003
}

\section{Conference Report by Karlheinz Steinmül- ler, Z_punkt GmbH Büro für Zukunftsgestal- tung, Berlin}

"Two days in the presence of the future," promised the invitation, "two days in the company of some of the finest future thinkers in Europe". In fact, the Global Future Forum (GFF) succeeded in bringing together about fifty future thinkers from European and North American companies, consultancies and the academic sphere to the Unisys International Management Center at St. Paul de Vence near Nice/France. Within the two days they discussed eight papers presented by GFF partners like Maria-Therese Hoppe (freelance futurologist from Denmark), Ian Vance (Amazing Communications Ltd, UK), and John Petersen (Arlington Institute, USA). On a third day, participants were able to join four topical workshops.

Founded just two years ago, the Global Future Forum (http://www.thegff.com) is an organisation that brings together up-to-date future-thinking to inject it into the wider business world. Consisting of an independent partnership of futurologists, management gurus, academics and business people, the GFF has been formed to help organisations extend their strategic planning horizons and better prepare for the future. In order to develop a comprehensive view of the total business environment over the next five to ten years, the GFF commissions strands of research to gauge the expectations of leading "thinkers" in various industry sectors. Twice or thrice a year, the GFF uses these panels of experts to achieve a collaborative judgment on certain scenarios, to analyse which are most likely and what level of impact they will have on a particular industry sector. In its "core agenda" the GFF investigates big global issues within the framework of several projects:

- the "Business Organisation Project" (about changes in management structures and responsibilities), 